\title{
AN EXPANSION THEOREM FOR A SYSTEM OF LINEAR DIFFEREN- TIAL EQUATIONS OF THE FIRST ORDER *
}

BY

\section{WALLIE ABRAHAM HURWITZ}

Introduction. The object of the present paper is to make a first step in the extension of the well-known Sturm-Liouville expansion theorem for a differential equation with one parameter to the case of systems of equations. A certain type of system of two equations of the first order in two unknowns will be considered. The essential facts deduced may be summarized in the following

THEOREM. The values of $\lambda$ for which the system

$$
\begin{aligned}
u^{\prime}(x)-[a(x)+\lambda] v(x) & =0, \\
v^{\prime}(x)+[b(x)+\lambda] u(x) & =0, \\
\alpha_{0} u(0)+\beta_{0} v(0) & =0, \\
\alpha_{1} u(1)+\beta_{1} v(1) & =0,
\end{aligned}
$$

(where $a(x), b(x)$ are continuous functions, $0 \leqq x \leqq 1$, and $\left|\alpha_{0}\right|+\left|\beta_{0}\right| \neq 0$, $\left.\left|\alpha_{1}\right|+\left|\beta_{1}\right| \neq 0\right)$ possesses non-trivial solutions, are all real, and infinite in number, extending to infinity positively and negatively and having no finite limitpoint. For each such value $\lambda_{n}$ there is one and only one solution $\left[u_{n}, v_{n}\right]$; the set of solutions corresponding to all values of $\lambda$ is orthogonal and may be normalized, these terms being used to characterize the relations:

$$
\int_{0}^{1}\left[u_{m} u_{n}+v_{m} v_{n}\right] d x= \begin{cases}0, & m \neq n, \\ 1, & m=n .\end{cases}
$$

If $f(x), g(x)$ are any two functions having continuous second derivatives ana satisfying the conditions

then

$$
\alpha_{0} f(0)+\beta_{0} g(0)=0, \quad \alpha_{1} f(1)+\beta_{1} g(1)=0,
$$

$$
\begin{aligned}
& f(x)=\sum_{n=-\infty}^{+\infty} c_{n} u_{n}(x), \\
& g(x)=\sum_{n=-\infty}^{+\infty} c_{n} v_{n}(x),
\end{aligned}
$$

* Presented to the Society, Sept. 5, 1917. 
where

$$
c_{n}=\int_{0}^{1}\left[f u_{n}+g v_{n}\right] d x,
$$

the convergence being uniform.

The method of treatment follows closely that of Kneser* on a single equation of the second order. A further extension of the present results to a more complicated form of boundary condition has been made by Dr. C. C. Camp.

It may be noted that the several systems of integral equations used are in each case of the Volterra type; solutions could therefore be obtained directly in the form of uniformly convergent infinite series without any use of the notation for the resolvent system to the kernel system; this notation serves only to abbreviate the presentation.

We deal with the homogeneous system of differential equations

$$
u^{\prime}-(a+\lambda) v=0, \quad v^{\prime}+(b+\lambda) u=0,
$$

and the corresponding non-homogeneous system

$$
x^{\prime}-(a+\lambda) v=\varphi, \quad v^{\prime}+(b+\lambda) u=\psi .
$$

Here the independent variable $x$ ranges over a finite real interval, which for convenience we take to be the interval $0 \leqq x \leqq 1 ; \lambda$ is a parameter which may have any real or complex value. The functions $a, b, \varphi, \psi$ are real and depend on $x$ alone; they are continuous, and the first two have continuous first derivatives. The unknown functions $u, v$ depend on $x$ and $\lambda$; the notation $u^{\prime}, v^{\prime}$ denotes differentiation with respect to $x$. Throughout the paper the variable $\lambda$ will be included in or omitted from the notation for function depending on it, as seems convenient.

A function-pair $[u, v]$, such that $u, v$ have continuous first derivatives and satisfy (1) or (2), will be called a solution; the product of a solution $[u, v]$ by a constant $c$ is to mean the solution $[c u, c v]$. The solution $[0,0]$ of (1) is a trivial solution.

We consider also the boundary conditions

$$
\begin{aligned}
& \alpha_{0} u(0)+\beta_{0} v(0)=0, \\
& \alpha_{1} u(1)+\beta_{1} v(1)=0,
\end{aligned}
$$

where $\alpha_{0}, \beta_{0}, \alpha_{1}, \beta_{1}$ are real constants such that

$$
\left|\alpha_{0}\right|+\left|\beta_{0}\right| \neq 0, \quad\left|\alpha_{1}\right|+\left|\beta_{1}\right| \neq 0 \text {. }
$$

In view of the latter conditions, there will be no loss of generality in writing, when this is convenient,

$$
\alpha_{0}=\cos \theta_{0}, \quad \beta_{0}=\sin \theta_{0} ; \quad \alpha_{1}=\cos \theta_{1}, \quad \beta_{1}=\sin \theta_{1} .
$$

* Mathematische Annalen, vol. 58 (1904), p. 81; vol. 60 (1905), p. 402. 
We shall use the notation $F=O(\varphi(\lambda))$, where $F$ is a function of $\lambda$ with or without $x$ and other real variables, to denote that there exist positive real constants $\Lambda, K$ such that $|F| \leqq K|\varphi|$ when $|\lambda|>\Lambda$; thus if $F$ depends on other variables besides $\lambda$, the notation involves the idea of uniformity. A similar notation will be used with a real (positive or negative) integer $n$ in place of $\lambda$.

The homogeneous system. THEOREM I. The system (1) with initial conditions

$$
u(0)=\alpha, \quad v(0)=\beta,
$$

where $\alpha, \beta$ are any constants, possesses for each $\lambda$ one and only one solution $[u, v]$; this solution is of the form

$$
\begin{aligned}
& u(x)=\alpha \cos \xi+\beta \sin \xi+o\left(\frac{1}{\lambda}\right), \\
& v(x)=\beta \cos \xi-\alpha \sin \xi+o\left(\frac{1}{\lambda}\right),
\end{aligned}
$$

where

$$
\xi=\lambda x+\frac{1}{2} \int_{0}^{x}[a(s)+b(s)] d s .
$$

Write

$$
\begin{aligned}
& u(x)=U(x)+\left(1+\frac{a}{2 \lambda}\right)(\alpha \cos \xi+\beta \sin \xi), \\
& v(x)=V(x)+\left(1+\frac{b}{2 \lambda}\right)(\beta \cos \xi-\alpha \sin \xi) .
\end{aligned}
$$

By substitution in (1) we find that

$$
\begin{aligned}
& U^{\prime}-(a+\lambda) V=\frac{P}{\lambda}, \\
& V^{\prime}+(b+\lambda) U=\frac{Q}{\lambda},
\end{aligned}
$$

where

$$
\begin{aligned}
& P(x, \lambda)=\frac{-\alpha a(b-a)-2 \beta a^{\prime}}{4} \sin \xi+\frac{\beta a(b-a)-2 \alpha a^{\prime}}{4} \cos \xi, \\
& Q(x, \lambda)=\frac{\alpha b(b-a)-2 \beta b^{\prime}}{4} \cos \xi+\frac{\beta b(b-a)+2 \alpha b^{\prime}}{4} \sin \xi,
\end{aligned}
$$

or

$$
\begin{aligned}
& P(x, \lambda)=\frac{a(b-a)}{4}(\beta \cos \xi-\alpha \sin \xi)-\frac{a^{\prime}}{2}(\alpha \cos \xi+\beta \sin \xi), \\
& Q(x, \lambda)=\frac{b(b-a)}{4}(\alpha \cos \xi+\beta \sin \xi)-\frac{b^{\prime}}{2}(\beta \cos \xi-\alpha \sin \xi),
\end{aligned}
$$


and that

$$
U(0)=-\frac{\alpha a(0)}{2 \lambda}, \quad V(0)=-\frac{\beta b(0)}{2 \lambda}
$$

If we multiply equations (8) respectively by $\cos \lambda x,-\sin \lambda x$, add, and integrate from 0 to $x$; and similarly multiply by $\sin \lambda x, \cos \lambda x$, add, and integrate, we obtain

(12) $U \cos \lambda x-V \sin \lambda x$

$$
=\int_{0}^{x}[a(s) V(s) \cos \lambda s+b(s) U(s) \sin \lambda s] d s+\frac{M}{\lambda},
$$

$U \sin \lambda x+V \cos \lambda x$

$$
=\int_{0}^{x}[a(s) V(s) \sin \lambda s-b(s) U(s) \cos \lambda s] d s+\frac{N}{\lambda},
$$

where

$$
\begin{aligned}
& M(x, \lambda)=-\frac{1}{2} \alpha a(0)+\int_{0}^{x}[P(s) \cos \lambda s-Q(s) \sin \lambda s] d s, \\
& N(x, \lambda)=-\frac{1}{2} \beta b(0)+\int_{0}^{x}[P(s) \sin \lambda s+Q(s) \cos \lambda s] d s .
\end{aligned}
$$

Now solving (12) algebraically for the $U, V$ appearing on the left, we have

$$
\begin{array}{r}
U=\frac{F}{\lambda}+\int_{0}^{x}[-b(s) U(s) \sin \lambda(x-s) \\
\quad+a(s) V(s) \cos \lambda(x-s)] d s, \\
V=\frac{G}{\lambda}+\int_{0}^{x}[-b(s) U(s) \cos \lambda(x-s) \\
\quad-a(s) V(s) \sin \lambda(x-s)] d s,
\end{array}
$$

where

$$
\begin{aligned}
& F(x, \lambda)=M \cos \lambda x+N \sin \lambda x, \\
& G(x, \lambda)=-M \sin \lambda x+N \cos \lambda x .
\end{aligned}
$$

This is a system of integral equations of Volterra type for $U, V$ :

$$
\begin{aligned}
& U(x)=\frac{F(x)}{\lambda}+\int_{0}^{x x}\left[K_{11}(x, s) U(s)+K_{12}(x, s) V(s)\right] d s, \\
& V(x)=\frac{G(x)}{\lambda}+\int_{0}^{x}\left[K_{21}(x, s) U(s)+K_{22}(x, s) V(s)\right] d s,
\end{aligned}
$$

where

$$
\begin{aligned}
& K_{11}(x, s, \lambda)=-b(s) \sin \lambda(x-s), \\
& K_{12}(x, s, \lambda)=a(s) \cos \lambda(x-s), \\
& K_{21}(x, s, \lambda)=-b(s) \cos \lambda(x-s), \\
& K_{22}(x, s, \lambda)=-a(s) \sin \lambda(x-s)
\end{aligned}
$$


To the kernel system $K_{i j}$ there exists a resolvent system $Q_{i j}$. The system (16) has the unique solution

$$
\begin{aligned}
& U(x)=\frac{F(x)}{\lambda}+\frac{1}{\lambda} \int_{0}^{x}\left[Q_{11}(x, s) F(s)+Q_{12}(x, s) G(s)\right] d s, \\
& V(x)=\frac{G(x)}{\lambda}+\frac{1}{\lambda} \int_{0}^{x}\left[Q_{21}(x, s) F(s)+Q_{22}(x, s) G(s)\right] d s .
\end{aligned}
$$

By (9),

hence by (13),

hence by (15),

and obviously by (17),

$$
P=O(1), \quad Q=O(1) ;
$$

$$
M=O(1), \quad N=O(1) ;
$$

$$
F=O(1), \quad G=O(1) ;
$$

$$
K_{i j}=O(1) \text {. }
$$

If we write out the infinite series for the functions $Q_{i j}$ of the resolvent system, analogous to the usual series of iterated kernels of a single kernel, it is clear that

$$
Q_{i j}=O(1) \text {. }
$$

Thus, finally, by (18), we see that

$$
U=O\left(\frac{1}{\lambda}\right), \quad V=O\left(\frac{1}{\lambda}\right)
$$

and this result substituted in (7) yields (5), and completes the proof of the theorem.

Furthermore, we have, from (9),

from (13),

$$
\partial P / \partial \lambda=O(1), \quad \partial Q / \partial \lambda=O(1) ;
$$

from (15),

$$
\partial M / \partial \lambda=O(1), \quad \partial N / \partial \lambda=O(1) ;
$$

and from (17),

$$
\partial F / \partial \lambda=O(1), \quad \partial G / \partial \lambda=O(1) ;
$$

Differentiating (16), we have

$$
\partial K_{i j} / \partial \lambda=O(1) .
$$

$$
\begin{aligned}
& \frac{\partial U(x)}{\partial \lambda}=\frac{S(x)}{\lambda}+\int_{0}^{x}\left[K_{11}(x, s) \frac{\partial U(s)}{\partial \lambda}+K_{12}(x, s) \frac{\partial V(s)}{\partial \lambda}\right] d s, \\
& \frac{\partial V(x)}{\partial \lambda}=\frac{T(x)}{\lambda}+\int_{0}^{x}\left[K_{21}(x, s) \frac{\partial U(s)}{\partial \lambda}+K_{22}(x, s) \frac{\partial V(s)}{\partial \lambda}\right] d s .
\end{aligned}
$$

where

$$
\begin{aligned}
& S(x)=\frac{\partial F(x)}{\partial \lambda}-\frac{F(x)}{\lambda}+\int_{0}^{x}\left[\frac{\partial K_{11}(x, s)}{\partial \lambda} U(s)+\frac{\partial K_{12}(x, s)}{\partial \lambda} V(s)\right] d s, \\
& T(x)=\frac{\partial G(x)}{\partial \lambda}-\frac{G(x)}{\lambda}+\int_{0}^{x}\left[\frac{\partial K_{21}(x, s)}{\partial \lambda} U(s)+\frac{\partial K_{22}(x, s)}{\partial \lambda} V(s)\right] d s .
\end{aligned}
$$


Evidently $S=O(1), T=O(1)$. Solving the integral system for $\partial U / \partial \lambda$, $\partial V / \partial \lambda$, we have

$$
\begin{aligned}
& \frac{\partial U(x)}{\partial \lambda}=\frac{S(x)}{\lambda}+\frac{1}{\lambda} \int_{0}^{x}\left[Q_{11}(x, s) S(s)+Q_{12}(x, s) T(s)\right] d s, \\
& \frac{\partial V(x)}{\partial \lambda}=\frac{T(x)}{\lambda}+\frac{1}{\lambda} \int_{0}^{x}\left[Q_{21}(x, s) S(s)+Q_{22}(x, s) T(s)\right] d s .
\end{aligned}
$$

Hence

$$
\frac{\partial U}{\partial \lambda}=O\left(\frac{1}{\lambda}\right), \quad \frac{\partial V}{\partial \lambda}=O\left(\frac{1}{\lambda}\right)
$$

Combining this result with (7), we have

TheoREM II. If. $[u, v]$ represents the solution of the system (1), (4), then

$$
\begin{aligned}
& \frac{\partial u(x)}{\partial \lambda}=x(\beta \cos \xi-\alpha \sin \xi)+O\left(\frac{1}{\lambda}\right), \\
& \frac{\partial v(x)}{\partial \lambda}=-x(\alpha \cos \xi+\beta \sin \xi)+O\left(\frac{1}{\lambda}\right) .
\end{aligned}
$$

Turning now to the system (1), $\left(B_{0}\right),\left(B_{1}\right)$, we seek first a solution of (1), $\left(B_{0}\right)$. Two such solutions for a single value of $\lambda$ are clearly linearly dependent; it will therefore suffice to choose a single non-trivial solution. Such a solution is obtained by using with (1) the initial conditions

$$
u(0)=-\beta_{0}=-\sin \theta_{0}, \quad v(0)=\alpha_{0}=\cos \theta_{0} .
$$

By Theorems I, II we have the

LemMn I. For every value of $\lambda$ a solution of $(1),\left(B_{0}\right)$ exists, and satisfies the conditions:

$$
\begin{gathered}
u(x)=\sin \left(\xi-\theta_{0}\right)+o\left(\frac{1}{\lambda}\right), \\
v(x)=\cos \left(\xi-\theta_{0}\right)+o\left(\frac{1}{\lambda}\right) \\
\frac{\partial u(x)}{\partial \lambda}=x \cos \left(\xi \cdots \theta_{0}\right)+o\left(\frac{1}{\lambda}\right), \\
\frac{\partial v(x)}{\partial \lambda}=-x \sin \left(\xi-\theta_{0}\right)+O\left(\frac{1}{\lambda}\right) .
\end{gathered}
$$

Any other solution of (1), ( $\left.B_{0}\right)$ for a fixed value of $\lambda$ is a constant multiple of this one.

It is now clear that a non-trivial solution of $(1),\left(B_{0}\right),\left(B_{1}\right)$ will exist only in case the solution just found for $(1),\left(B_{0}\right)$ satisfies also $\left(B_{1}\right)$; this gives a 
condition on $\lambda$. We define

$$
D(\lambda)=\alpha_{1} u(1)+\beta_{1} v(1)=u(1) \cos \theta_{1}+v(1) \sin \theta_{1},
$$

and note that

$$
\frac{d D(\lambda)}{d \lambda}=\frac{\partial u(1)}{\partial \lambda} \cos \theta_{1}+\frac{\partial v(1)}{\partial \lambda} \sin \theta_{1}
$$

Then the condition for a solution of $(1),\left(B_{0}\right)$ to satisfy also $\left(B_{1}\right)$ is that

$$
D(\lambda)=0 .
$$

If we now evaluate $D(\lambda), d D(\lambda) / d \lambda$ by use of (21), we find that

$$
\begin{gathered}
D(\lambda)=\sin \left(\xi_{1}+\theta_{1}-\theta_{0}\right)+o\left(\frac{1}{\lambda}\right), \\
\frac{d D(\lambda)}{d \lambda}=\cos \left(\xi_{1}+\theta_{1}-\theta_{0}\right)+o\left(\frac{1}{\lambda}\right),
\end{gathered}
$$

where

$$
\xi_{1}=\xi(1)=\lambda+\frac{1}{2} \int_{0}^{1}[a(s)+b(s)] d s,
$$

or, if we write $\frac{1}{2} \int_{0}^{1}[a(s)+b(s)] d s+\theta_{1}-\theta_{0}=-\theta$,

$$
\begin{gathered}
D(\lambda)=\sin (\lambda-\theta)+o\left(\frac{1}{\lambda}\right), \\
\frac{d D(\lambda)}{d \lambda}=\cos (\lambda-\theta)+o\left(\frac{1}{\lambda}\right) .
\end{gathered}
$$

In order to investigate the roots of (23), we first prove

LEMMA II. If $\left[u_{1}, v_{1}\right],\left[u_{2}, v_{2}\right]$ represent solutions of $(1),\left(B_{0}\right),\left(B_{1}\right)$ for two distinct values $\lambda_{1}, \lambda_{2}$ respectively of $\lambda$, then

$$
\int_{0}^{1}\left[u_{1} u_{2}+v_{1} v_{2}\right] d x=0
$$

If we multiply the equations

$$
\begin{aligned}
u_{1}^{\prime}-\left(a+\lambda_{1}\right) v_{1} & =0, \\
v_{1}^{\prime}+\left(b+\lambda_{1}\right) u_{1} & =0, \\
u_{2}^{\prime}-\left(a+\lambda_{2}\right) v_{2} & =0, \\
v_{2}^{\prime}+\left(b+\lambda_{2}\right) u_{2} & =0,
\end{aligned}
$$

respectively by $v_{2},-u_{2},-v_{1}, u_{1}$, and add, we have

$$
\frac{d}{d x}\left[u_{1} v_{2}-u_{2} v_{1}\right]=\left(\lambda_{1}-\lambda_{2}\right)\left[u_{1} u_{2}+v_{1} v_{2}\right]
$$


hence

$$
\left(\lambda_{1}-\lambda_{2}\right) \int_{0}^{1}\left[u_{1} u_{2}+v_{1} v_{2}\right] d x=\left[u_{1} v_{2}-u_{2} v_{1}\right]_{0}^{1}
$$

But since both solutions satisfy $\left(B_{0}\right),\left(B_{1}\right)$, the expression on the right vanishes. Therefore if $\lambda_{1} \neq \lambda_{2}$, the integral on the left must vanish.

From this result we deduce

LEMma III. $D(\lambda)$ has only real roots.

If $\lambda$ is a root, to which corresponds the non-trivial solution $[u, v]$, then we see by inspection of $(1),\left(B_{0}\right),\left(B_{1}\right)$ that $\bar{\lambda}$ must also be a root with the solution $[\bar{u}, \bar{v}]$. If $\lambda, \bar{\lambda}$ were distinct, then by Lemma II,

$$
\int_{0}^{1}[u \bar{u}+v \bar{v}] d x=0
$$

but this would imply that $u=v=0$, and the solution would be trivial. Hence $\lambda=\bar{\lambda}$, and $\lambda$ must be real.

We wish to show also that no root of $D(\lambda)$ can be double. For a double root we should have simultaneously

$$
\begin{gathered}
D(\lambda)=\alpha_{1} u(1)+\beta_{1} v(1)=0 \\
\frac{d D(\lambda)}{d \lambda}=\alpha_{1} \frac{\partial u(1)}{\partial \lambda}+\beta_{1} \frac{\partial v(1)}{\partial \lambda}=0
\end{gathered}
$$

Hence it would follow that

$$
u(1) \frac{\partial v(1)}{\partial \lambda}-v(1) \frac{\partial u(1)}{\partial \lambda}=0
$$

If however we multiply the equations

$$
\begin{aligned}
u^{\prime}-(a+\lambda) v & =0, \\
v^{\prime}+(b+\lambda) u & =0, \\
\left(\frac{\partial u}{\partial \lambda}\right)^{\prime}-(a+\lambda) \frac{\partial v}{\partial \lambda} & =v, \\
\left(\frac{\partial v}{\partial \lambda}\right)^{\prime}+(b+\lambda) \frac{\partial u}{\partial \lambda} & =-u,
\end{aligned}
$$

(of which the last pair are obvious consequences of the first pair) respectively by $\partial v / \partial \lambda,-\partial u / \partial \lambda,-v, u$, add, and integrate from 0 to 1 , we find

$$
\left[u \frac{\partial v}{\partial \lambda}-v \frac{\partial u}{\partial \lambda}\right]_{0}^{1}=-\int_{0}^{1}\left[u^{2}+v^{2}\right] d x,
$$

which, since $\partial u / \partial \lambda$ and $\partial v / \partial \lambda$ vanish at $x=0$, reduces to

$$
-\int_{0}^{1}\left[u^{2}+v^{2}\right] d x=u(1) \frac{\partial v(1)}{\partial \lambda}-v(1) \frac{\partial u(1)}{\partial \lambda}=0,
$$


by (30). But from this it would follow that $u$ and $v$ are identically zero, which is untrue. Hence

LEMмA IV. $D(\lambda)$ has only simple roots.

In order to determine the location of the real roots, we divide the whole range of real values of $\lambda$ into two sets of intervals:

$$
\begin{aligned}
& I_{n}:|\lambda-\theta-n \pi| \leqq \frac{\pi}{4}, \\
& J_{n}:\left|\lambda-\theta-\left(n+\frac{1}{2}\right) \pi\right| \leqq \frac{\pi}{4},
\end{aligned}
$$

where $n$ is any integer. At the beginning of any interval $I_{n}$,

and by (27),

$$
\lambda=\theta+\left(n-\frac{1}{4}\right) \pi,
$$

$$
\begin{aligned}
D(\lambda) & =\sin \left(n-\frac{1}{4}\right) \pi+O\left(\frac{1}{\lambda}\right) \\
& =\frac{(-1)^{n-1}}{\sqrt{2}}+O\left(\frac{1}{\lambda}\right) .
\end{aligned}
$$

Similarly at the end of an interval $I_{n}, \lambda=\theta+\left(n+\frac{1}{4}\right) \pi$, and

$$
D(\lambda)=\frac{(-1)^{n}}{\sqrt{2}}+O\left(\frac{1}{\lambda}\right)
$$

For sufficiently large $|\lambda|$, therefore, $D(\lambda)$ has opposite signs at the beginning and end of $I_{n}$, and hence vanishes at least once inside $I_{n}$.

In any interval $J_{n} . \lambda=\theta+\left(n+\frac{1}{2}\right) \pi+\delta,|\delta| \leqq \pi / 4$, and

$$
D(\lambda)=(-1)^{n} \cos \delta+O\left(\frac{1}{\lambda}\right) \text {. }
$$

But since $|\cos \delta| \geqq 1 / \sqrt{2}$, it follows that for sufficiently large $|\lambda|, D(\lambda) \neq 0$ throughout $J_{n}$.

An argument analogous to the precediny shows that for sufficiently large $|\lambda|, d D(\lambda) / d \lambda \neq 0$ throughout $I_{n}$. Therefore $D(\lambda)$ cannot have more than one root in each $I_{n}$.

Having now proved that for sufficiently large $|\lambda|, D(\lambda)$ has one and only one root in each $I_{n}$ and none outside $I_{n}$, we may write for the large roots

$$
\lambda_{n}=\theta+n \pi+\epsilon_{n}, \quad\left|\epsilon_{n}\right| \leqq \frac{\pi}{4} .
$$

This expression, substituted in (27), gives

$$
0=(-1)^{n} \sin \epsilon_{n}+O\left(\frac{1}{n}\right),
$$


or

$$
\sin \epsilon_{n}=O\left(\frac{1}{n}\right)
$$

Since the choice among the angles $\epsilon_{n}$ corresponding to a given $\sin \epsilon_{n}$ is here uniquely determined by the condition $\left|\epsilon_{n}\right| \leqq \pi / 4$, we have

and finally from (32),

$$
\epsilon_{n}=O\left(\frac{1}{n}\right)
$$

$$
\lambda_{n}=\theta+n \pi+O\left(\frac{1}{n}\right) .
$$

By using this formula for $\lambda_{n}$ in (21), we find for the solution $\left[u_{n}, v_{n}\right]$ corresponding to the root $\lambda_{n}$ of $D(\lambda)$,

$$
\begin{aligned}
& u_{n}(x)=\sin \left(\xi_{n}-\theta_{0}\right)+o\left(\frac{1}{n}\right), \\
& v_{n}(x)=\cos \left(\xi_{n}-\theta_{0}\right)+o\left(\frac{1}{n}\right),
\end{aligned}
$$

where $\xi_{n}$ is the value of $\xi$ for $\lambda=\lambda_{n}$.

We have seen that two solutions $\left[u_{m}, v_{m}\right],\left[u_{n}, v_{n}\right]$ are in a general sense orthogonal; that is,

$$
\int_{0}^{1}\left[u_{m} u_{n}+v_{m} v_{n}\right] d x=0
$$

We now seek to have each solution normalized:

$$
\int_{0}^{1}\left[u_{n}^{2}+v_{n}^{2}\right] d x=1 .
$$

This can be accomplished by dividing $u_{n}$ and $v_{n}$ by the constant

$$
\sqrt{\int_{0}^{1}\left[u_{n}^{2}+v_{n}^{2}\right] d x} .
$$

But it is clear from the expressions just found for $u_{n}, v_{n}$, that

$$
\int_{0}^{1}\left[u_{n}^{2}+v_{n}^{2}\right] d x=1+O\left(\frac{1}{n}\right) ;
$$

hence normalization leaves the asymptotic forms for $u_{n}(x), v_{n}(x)$ unchanged. We shall continue to use the same notation for the normalized solutions.

We collect the preceding results as follows:

Theовем III. The roots of $D(\lambda)$ are real and simple. For sufficiently large valies of $|\lambda|$ they have the asymptotic expression

$$
\lambda_{n}=\theta+n \pi+o\left(\frac{1}{n}\right)
$$


$n$ being an integer. To each root corresponds one and (except for multiplication by a constant) only one solution $\left[u_{n}, v_{n}\right]$ of $(1),\left(B_{0}\right),\left(B_{1}\right)$. These solutions may be taken to form a normal orthogonal set

$$
\int_{0}^{1}\left[u_{m} u_{n}+v_{m} v_{n}\right] d x=\left\{\begin{array}{l}
0, m \neq n, \\
1, m=n
\end{array}\right.
$$

and have the asymptotic expressions

$$
\begin{aligned}
& u_{n}(x)=\sin \left(\xi_{n}-\theta_{0}\right)+o\left(\frac{1}{n}\right), \\
& v_{n}(x)=\cos \left(\xi_{n}-\theta_{0}\right)+O\left(\frac{1}{n}\right) .
\end{aligned}
$$

The non-homogeneous system. Consider now the system (2). Let $\left[u_{0}, v_{0}\right]$ denote a solution satisfying the initial conditions

$$
u_{0}(0)=0, \quad v_{0}(0)=0 .
$$

We call $\left[u_{1}, v_{1}\right]$ the previously discussed solution of (1) satisfying the initial conditions

$$
u_{1}(0)=-\sin \theta_{0}, \quad v_{1}(0)=\cos \theta_{0},
$$

and introduce a second solution $\left[u_{2}, v_{2}\right]$ of (1) satisfying the initial conditions

$$
u_{2}(0)=\cos \theta_{0}, \quad v_{2}(0)=\sin \theta_{0} .
$$

Each of the six functions $u_{0}, v_{0}, u_{1}, v_{1}, u_{2}, v_{2}$ is an integral function of the complex variable $\lambda$. The solutions $\left[u_{1}, v_{1}\right],\left[u_{2}, v_{2}\right]$ are linearly independent, since

$$
\left|\begin{array}{ll}
u_{1}(0) & v_{1}(0) \\
u_{2}(0) & v_{2}(0)
\end{array}\right|=-1 \neq 0
$$

Recalling the notation

we also write

$$
D(\lambda)=u_{1}(1) \cos \theta_{1}+v_{1}(1) \sin \theta_{1},
$$

$$
D_{0}(\lambda)=u_{0}(1) \cos \theta_{1}+v_{0}(1) \sin \theta_{1} .
$$

Both $D(\lambda)$ and $D_{0}(\lambda)$ are clearly integral functions of $\lambda$.

Any solution of (2) can be written in the form

$$
\begin{aligned}
& u=u_{0}+c_{1} u_{1}+c_{2} u_{2}, \\
& v=v_{0}+c_{1} v_{1}+c_{2} v_{2},
\end{aligned}
$$

by proper choice of the numbers $c_{1}, c_{2}$, which are independent of $x$, but vary with $\lambda$. We seek to choose $c_{1}, c_{2}$ so as to satisfy $\left(B_{0}\right),\left(B_{1}\right)$. For $\left(B_{0}\right)$ we need merely take $c_{2}=0$. Placing this value in (40) and writing out 
$\left(B_{2}\right)$, we have

$$
D_{0}(\lambda)+c_{1} D(\lambda)=0 .
$$

In case $D(\lambda) \neq 0$, we see that

$$
c_{1}=-\frac{D_{0}(\lambda)}{D(\lambda)}
$$

and hence find for $(2),\left(B_{0}\right),\left(B_{1}\right)$ the unique solution

$$
\begin{aligned}
& u=u_{0}-\frac{D_{0}(\lambda)}{D_{1}(\lambda)} u_{1}, \\
& v=v_{0}-\frac{D_{0}(\lambda)}{D_{1}(\lambda)} v_{1} .
\end{aligned}
$$

Thus we may state

Lemma V. If $D(\lambda) \neq 0$, the system $(2),\left(B_{0}\right),\left(B_{1}\right)$ has one and only one solution $[u, v]$. Throughout any region in which $D(\lambda) \neq 0, u$ and $v$ are analytic functions of $\lambda$.

If however $\lambda$ has one of the values $\lambda_{n}$, so that $D(\lambda)=0$, then (37) cannot be satisfied for any $c_{1}$ unless also $D_{0}(\lambda)=0$, and is satisfied for every $c_{1}$ in case $D_{0}(\lambda)=0$. Let us examine the meaning of the simultaneous vanishing of $D(\lambda), D_{0}(\lambda)$. Writing out (2) for $\left[u_{0}, v_{0}\right]$ and (1) for $\left[u_{1}, v_{1}\right]$, multiplying the four equations in order by $v_{1},-u_{1},-v_{0}, u_{0}$, adding, and integrating from 0 to 1 , we have

But if

$$
u_{0}(1) v_{1}(1)-v_{0}(1) u_{1}(1)=\int_{0}^{1}\left[\varphi v_{1}-\psi u_{1}\right] d x
$$

then

and hence

$$
\begin{aligned}
& D(\lambda)=u_{1}(1) \cos \theta_{1}+v_{1}(1) \sin \theta_{1}=0, \\
& D_{0}(\lambda)=u_{0}(1) \cos \theta_{1}+v_{0}(1) \sin \theta_{1}=0,
\end{aligned}
$$

$$
u_{0}(1) v_{1}(1)-v_{0}(1) u_{1}(1)=0,
$$

$$
\int_{0}^{1}\left[\varphi v_{1}-\psi u_{1}\right] d x=0,
$$

or, since $\left[u_{1}, v_{1}\right]$ here represents the solution $\left[u_{n}, v_{n}\right]$ corresponding to the $\operatorname{root} \lambda_{n}$ of $D(\lambda)$,

$$
\int_{0}^{1}\left[\varphi v_{n}-\psi \cdot u_{n}\right] d x=0
$$

Conversely, if this condition is satisfied and $D(\lambda)=0$, then $D_{0}(\lambda)=0$. Thus when $D(\lambda)=0,(43)$ is a necessary and sufficient condition that also $D_{0}(\lambda)=0$.

If then (43) holds, the system (2), $\left(B_{0}\right),\left(B_{1}\right)$ still has solutions for $\lambda=\lambda_{n}$, 
which are of the form

$$
\begin{aligned}
u & =u_{0}+c_{1} u_{n}, \\
v & =v_{0}+c_{1} v_{n},
\end{aligned}
$$

for any $c_{1}$. We inquire whether a particular $c_{1}$ can be chosen in such a way that the solution $[u, v]$ of $(2),\left(B_{0}\right),\left(B_{1}\right)$ for neighboring values of $\lambda$ shall join analytically to the solution for $\lambda_{n}$. Since $u_{0}, v_{0}, u_{1}, v_{1}$ are analytic for all $\lambda$, we see from (42) that it is sufficient that the quotient $D_{0}(\lambda) / D(\lambda)$ approach a limit as $\lambda$ approaches $\lambda_{n}$. This will always occur:

$$
\lim _{\lambda \rightarrow \lambda_{n}} \frac{D_{0}(\lambda)}{D(\lambda)}=\frac{D_{0}^{\prime}\left(\lambda_{n}\right)}{D^{\prime}\left(\lambda_{n}\right)}
$$

since $D^{\prime}\left(\lambda_{n}\right) \neq 0$ by Lemma IV. The resulting [ $u, v$ ], being analytic in $\lambda$ near $\lambda_{n}$ and continuous at $\lambda_{n}$, will be analytic also at $\lambda_{n}$. Thus we have if

Lemma VI. For $\lambda=\lambda_{n}$, the system $(2),\left(B_{0}\right),\left(B_{1}\right)$ has solutions if and only

$$
\int_{0}^{1}\left[\varphi v_{n}-\psi u_{n}\right] d x=0 .
$$

If this condition is satisfied, a solution $[u, v]$ can be selected which joins with the unique solution for neighboring values of $\lambda$ in such $a$ way that $u$ and $v$ are analytic throughout a region surrounding $\lambda_{n}$.

From this result we have further

LEMMA VII. If for every $\lambda_{n}$,

$$
\int_{0}^{1}\left[\varphi v_{n}-\psi u_{n}\right] d x=0,
$$

then the system $(2),\left(B_{0}\right),\left(B_{1}\right)$ possesses a solution $[u, v]$ such that $u$ and $v$ are integral functions of $\lambda$.

Let us now interpret further the meaning of the condition just obtained. Suppose that (43) holds for each $\lambda_{n}$. Then the solution $[u, v]$ of $(2),\left(B_{0}\right)$, $\left(B_{1}\right)$, being analytic for all $\lambda$ by Lemma VII, can be written

$$
\begin{aligned}
u & =\sum_{n=0}^{\infty} \lambda^{n} y_{n}(x), \\
v & =\sum_{n=0}^{\infty} \lambda^{n} z_{n}(x),
\end{aligned}
$$

where the series converge, uniformly in $x$, for all $\lambda$ in the complex plane and for $0 \leqq x \leqq 1$. It is easily seen that $y_{n}, z_{n}$ are in all cases real. Substituting in (2) and equating coefficients, we have

$$
\begin{aligned}
& y_{n}^{\prime}-a z_{n}=z_{n-1}, \\
& z_{n}^{\prime}+b y_{n}=-y_{n-1}
\end{aligned}
$$


for all values of $n$, provided we agree to define

$$
y_{-1}=-\psi, \quad z_{-1}=\varphi \text {. }
$$

Write (45) and a similar pair of equations obtained by putting $m$ for $n$; multiply these four equations respectively by $z_{m},-y_{m},-z_{n}, y_{n}$, add, and integrate from 0 to 1 ; we have

$$
\int_{0}^{1}\left[y_{m-1} y_{n}+z_{m-1} z_{n}\right] d x=\int_{0}^{1}\left(y_{m} y_{n-1}+z_{m} z_{n-1}\right] d x
$$

By repeated application of this result it is seen that if we define

$$
W_{r}=\int_{0}^{1}\left[y_{0} y_{r}+z_{0} z_{r}\right] d x
$$

then

$$
\int_{0}^{1}\left[y_{m} y_{n}+z_{m} z_{n}\right] d x=W_{m+n}
$$

Integrating the inequality

$$
\begin{aligned}
& \left.\begin{array}{ll}
y_{m+1}(x) & y_{m+1}(\xi) \\
y_{m-1}(x) & y_{m-1}(\xi)
\end{array}\right|^{2}+\left|\begin{array}{ll}
y_{m+1}(x) & z_{m+1}(\xi) \\
y_{m-1}(x) & z_{m-1}(\xi)
\end{array}\right|^{2} \\
& +\left|\begin{array}{ll}
z_{m+1}(x) & y_{m+1}(\xi) \\
z_{m-1}(x) & y_{m-1}(\xi)
\end{array}\right|^{2}+\left|\begin{array}{ll}
z_{m+1}(x) & z_{m+1}(\xi) \\
z_{m-1}(x) & z_{m-1}(\xi)
\end{array}\right|^{2} \geqq 0
\end{aligned}
$$

with respect to both $x$ and $\xi$ between the limits 0 and 1 , we have

$$
W_{2 m-2} W_{2 m+2}-W_{2 m}^{2} \geqq 0 \text {. }
$$

If we multiply (44) respectively by $y_{0}, z_{0}$ and integrate from 0 to 1 , we have the series

$$
\sum_{n=0}^{\infty} W_{n} \lambda^{n}
$$

convergent for all $\lambda$. Omitting alternate terms, we have the series

$$
W_{0}+\lambda^{2} W_{2}+\lambda^{4} W_{4}+\cdots,
$$

also (since the convergence of a power series is absolute) convergent for all $\lambda$. By (48),

$$
W_{2 m}=\int_{0}^{1}\left[y_{m}^{2}+z_{m}^{2}\right] d x \geqq 0 \text {. }
$$

We shall show that some $W_{2 m}=0$. If $W_{2 m} \neq 0$ for all $m$, then by (48), $W_{2 m}>0$, and by (49),

$$
\frac{W_{2 m-2}}{W_{2 m}} \geqq \frac{W_{2 m}}{W_{2 m+2}}
$$


Hence

$$
\frac{W_{0}}{W_{2}} \geqq \frac{W_{2}}{W_{4}} \geqq \frac{W_{4}}{W_{6}} \geqq \cdots
$$

Therefore the series (50) could not converge for $\lambda=\sqrt{W_{0} / W_{2}}$, since for that value the terms of (50) would be respectively greater numerically than the terms of the series

$$
W_{0}+W_{0}+W_{0}+\cdots
$$

Thus, for some $m, W_{2 m}=0$; that is, by (48),

$$
\int_{0}^{1}\left[y_{m}^{2}+z_{m}^{2}\right] d x=0
$$

whence

$$
y_{m}=z_{m}=0
$$

and, by repeated applications of (45),

Thus we have

$$
\begin{aligned}
& y_{m-1}=z_{m-1}=0, \\
& y_{m-2}=z_{m-2}=0, \\
& \vdots \cdot \cdot \cdot \cdot \\
& y_{0}=z_{0}=0, \\
& \varphi=\psi=0 .
\end{aligned}
$$

Theorem IV. If $\varphi(x), \psi(x)$ are continuous functions such that for every solution $\left[u_{n}, v_{n}\right]$ of $(1),\left(B_{0}\right),\left(B_{1}\right)$,

$$
\int_{n}^{1}\left[\varphi v_{n}-\psi u_{n}\right] d x=0
$$

then

$$
\varphi=\psi=0 \text {. }
$$

Expansion of an arbitrary function-pair. Let $f(x), g(x)$ be a pair of continuous functions, and let there exist the simultaneous expansions

$$
\begin{aligned}
& f(x)=\sum_{n=-\infty}^{+\infty} c_{n} u_{n}(x), \\
& g(x)=\sum_{n=-\infty}^{+\infty} c_{n} v_{n}(x) .
\end{aligned}
$$

If both series converge uniformly, then the coefficients can be determined by multiplying respectively by $u_{n}(x), v_{n}(x)$, adding, and integrating from 0 to 1. In view of the normal orthogonality of the set of $\left[u_{n}, v_{n}\right]$, this process gives

$$
c_{n}=\int_{0}^{1}\left[f u_{n}+g v_{n}\right] d x
$$


If term-wise differentiation of (51) is permissible, it is clear that $f, g$ must satisfy also the boundary conditions

$$
\begin{aligned}
& \alpha_{0} f(0)+\beta_{0} g(0)=0, \\
& \alpha_{1} f(1)+\beta_{1} g(1)=0 .
\end{aligned}
$$

We now proceed to investigate the convergence of the two series (51), in which the coefficients are determined by (52) in terms of two given functions $f(x), g(x)$. It is assumed that $f$ and $g$ have continuous second derivatives. Using (1), and integrating by parts twice, we have

$$
\begin{aligned}
\int_{0}^{1} f u_{n} d x & =-\int_{0}^{1} \frac{f v_{n}^{\prime}}{\lambda_{n}+b} d x \\
& =\left[-\frac{f v_{n}}{\lambda_{n}+b}\right]_{0}^{1}+\int_{0}^{1} v_{n} \frac{d}{d x} \frac{f}{\lambda_{n}+b} d x \\
& =\left[-\frac{f v_{n}}{\lambda_{n}+b}\right]_{0}^{1}+\int_{0}^{1} \frac{u_{n}^{\prime}}{\lambda_{n}+a} \frac{d}{d x} \frac{f}{\lambda_{n}+b} d x \\
& =\left[-\frac{f v_{n}}{\lambda_{n}+b}+\frac{u_{n}}{\lambda_{n}+a} \frac{d}{d x} \frac{f}{\lambda_{n}+b}\right]_{0}^{1}-\int_{0}^{1} u_{n} \frac{d}{d x}\left\{\frac{1}{\lambda_{n}+a} \frac{d}{d x} \frac{f}{\lambda_{n}+b}\right\} d x .
\end{aligned}
$$

Similarly,

$$
\begin{aligned}
\int_{0}^{1} g v_{n} d x=\left[\frac{g u_{n}}{\lambda_{n}+a}+\frac{v_{n}}{\lambda_{n}+b} \frac{d}{d x} \frac{g}{\lambda_{n}+\bar{a}}\right]_{0}^{1} & \\
& -\int_{0}^{1} v_{n} \frac{d}{d x}\left\{\frac{1}{\lambda_{n}+b} \frac{d}{d x} \frac{g}{\lambda_{n}+a}\right\} d x .
\end{aligned}
$$

By direct computation, we find that

$$
\begin{aligned}
& \frac{1}{\lambda_{n}+a} \frac{d}{d x} \frac{f}{\lambda_{n}+b}=O\left(\frac{1}{\lambda_{n}^{2}}\right)=O\left(\frac{1}{n^{2}}\right), \\
& \frac{1}{\lambda_{n}+b} \frac{d}{d x} \frac{g}{\lambda_{n}+a}=O\left(\frac{1}{\lambda_{n}^{2}}\right)=. O\left(\frac{1}{n^{2}}\right), \\
& \frac{d}{d x}\left\{\frac{1}{\lambda_{n}+a} \frac{d}{d x} \frac{f}{\lambda_{n}+b}\right\}=O\left(\frac{1}{\lambda_{n}^{2}}\right)=O\left(\frac{1}{n^{2}}\right), \\
& \frac{d}{d x}\left\{\frac{1}{\lambda_{n}+b} \frac{d}{d x} \frac{g}{\lambda_{n}+a}\right\}=O\left(\frac{1}{\lambda_{n}^{2}}\right)=O\left(\frac{1}{n^{2}}\right) .
\end{aligned}
$$

Hence

$$
\begin{aligned}
& \int_{0}^{1} f u_{n} d x=\left[-\frac{f v_{n}}{\lambda_{n}+b}\right]_{0}^{1}+O\left(\frac{1}{n^{2}}\right), \\
& \int_{0}^{1} g v_{n} d x=\left[\frac{g u_{n}}{\lambda_{n}+a}\right]_{0}^{1}+O\left(\frac{1}{n^{2}}\right),
\end{aligned}
$$


and

But

$$
c_{n}=\left[-\frac{f v_{n}}{\lambda_{n}+b}+\frac{g u_{n}}{\lambda_{n}+a}\right]_{0}^{1}+O\left(\frac{1}{n^{2}}\right) .
$$

and

$$
-\frac{f v_{n}}{\lambda_{n}+b}+\frac{g u_{n}}{\lambda_{n}+a}=\frac{-f v_{n}+g u_{n}}{\lambda_{n}}+O\left(\frac{1}{n^{2}}\right)
$$

$$
c_{n}=\left[-\frac{f v_{n}+g u_{n}}{\lambda_{n}}\right]_{0}^{1}+O\left(\frac{1}{n^{2}}\right)
$$

In case $f, g$ satisfy $\left(C_{0}\right),\left(C_{1}\right)$,

and

$$
\left[-f v_{n}+g u_{n}\right]_{0}^{1}=0,
$$

$$
c_{n}=O\left(\frac{1}{n^{2}}\right)
$$

As the series

$$
\sum_{n=1}^{\infty} \frac{1}{n^{2}}
$$

converges, and as

$$
u_{n}=O(1), \quad v_{n}=O(1),
$$

both the series (51) converge uniformly. It remains to be seen whether they represent $f$ and $g$. Write

$$
\begin{aligned}
& F(x)=\sum_{n=-\infty}^{+\infty} c_{n} u_{n}(x), \\
& G(x)=\sum_{n=-\infty}^{+\infty} c_{n} v_{n}(x) .
\end{aligned}
$$

From (53),

while also by (52),

$$
c_{n}=\int_{0}^{1}\left[F u_{n}+G v_{n}\right] d x
$$

$$
c_{n}=\int_{0}^{1}\left[f u_{n}+g v_{n}\right] d x
$$

Hence, subtracting,

$$
\int_{0}^{1}\left[(F-f) u_{n}+(G-g) v_{n}\right] d x=0,
$$

and by Theorem IV,

$$
F=f, \quad G=g \text {. }
$$

Two details which have been neglected in the above proof require further consideration. In the first place, the use of $\lambda_{n}+a, \lambda_{n}+b$ in denominators is permissible, since for sufficiently large $\lambda_{n}$, they do not vanish. Secondly, $u_{n}, v_{n}$ have been used above with the understanding that the subscript denotes 
the serial order of the solution in question, while in the earlier part of the paper, $u_{n}, v_{n}$ have indicated the solution corresponding to the root $\lambda_{n}$ of $D(\lambda)$ lying in $I_{n}$. Since for sufficiently large $\lambda$ one and only one root of $D(\lambda)$ lies in each $I_{n}$, the two interpretations of the subscript differ only by a constant, so that the evaluation $\lambda_{n}=O(n)$ is still correct. We have thus finally proved

TheOREM V. If $f(x), g(x)$ are any two functions possessing continuous second derivatives, $0 \leqq x \leqq 1$, and satisfying the boundary conditions $\left(C_{0}\right)$, $\left(C_{1}\right)$, then they may be simultaneously expanded in the uniformly convergent series:

where

$$
\begin{aligned}
& f(x)=\sum_{n=-\infty}^{+\infty} c_{n} u_{n}(x), \\
& g(x)=\sum_{n=-\infty}^{+\infty} c_{n} v_{n}(x),
\end{aligned}
$$

CORNELL UNIVERSTTY,

$$
c_{n}=\int_{0}^{1}\left[f u_{n}+g v_{n}\right] d x .
$$

ITHACA, N. Y.

\section{Author's CoRrection}

0. E. Glenn. A memoir upon formal invariancy with regard to binary modular transformations. Invariants of relativity ( $\mathrm{Tr}$ a $\mathrm{n} \mathrm{s}$ a c t i o $\mathrm{n}$, vol. 21, pp. 285312 ).

(1) My attention was directed by Dr. W. L. G. Williams to the existence of a certain seminvariant of $f_{4}$ modulo 3 , of degree four, which is not reducible in terms of the nine seminvariants given on page 295 and stated there, in a theorem, to form a complete system. The seminvariant is of a type for which the starred assumption on page 296 is not valid. The error makes my result for the seminvariants of the quartic modulo 3 much less general than is indicated in the theorem. However, when the system is completed by discovery of the requisite new forms my starred assumption can perhaps be proved for it, and thus my theory would be completed.

(2) The following quadratic covariant was inadvertently omitted from the lists (35), (40), (47):

$$
g=s x_{1}^{2}+\left(s+b_{0} b_{1}+b_{0}^{2}\right) x_{1} x_{2}+b_{0}\left(b_{0}+b_{1}+b_{2}\right) x_{2}^{2} .
$$

